\title{
Use of a Y-Shaped Plate for Intermaxillary Fixation
}

\author{
Tae Hoon Kim ${ }^{1}$ \\ Il Hyung Yang ${ }^{2}$, \\ Kyung Won Minn', \\ Ung Sik Jin ${ }^{1}$ \\ ${ }^{1}$ Department of Plastic and Reconstructive \\ Surgery, Seoul National University College \\ of Medicine, Seoul; ${ }^{2}$ Department of \\ Orthodontics, Seoul National University \\ Dental Hospital, Seoul, Korea
}

No potential conflict of interest relevant to this article was reported.

\begin{abstract}
Maxillomandibular fractures usually require intermaxillary fixation as a means to immobilize and stabilize the fracture and to re-establish proper occlusion. Arch bars or intermaxillary fixation screws cannot be used for edentulous patients or for patients who have poor dental health. Here, we present a case of repeated intermaxillary fixation failure in a patient weak alveolar rigidity secondary to multiple dental implants. Because single-point fixation screws were not strong enough to maintain proper occlusion, we have used $Y$-shaped plates to provide more rigid anchoring points for the intermaxillary wires. We suggest that this method should be considered for patients in whom conventional fixation methods are inappropriate or have failed.
\end{abstract}

Keywords: Mandible / Jaw fixation techniques / Titanium plate / Mandibular reconstruction

\section{INTRODUCTION}

Maxillomandibular fractures usually require intermaxillary fixation as a means to immobilize and stabilize the fracture and to reestablish proper occlusion. Various techniques for intermaxillary fixation have been described in the literature. Traditionally, intermaxillary fixation has been carried out with the use of wires applied directly on interdentitial space or to arch bars. More recently, intermaxillary fixation screws have been used successfully. However, arch bars or intermaxillary fixation screws cannot be used for edentulous patients or for patients who have poor dental health. Here, we introduce an alternative technique of intermaxillary fixation using a Y-shaped plate.

\section{CASE REPORT}

A 54-year-old man was injured in a car accident and was subse-

\section{Correspondence: Ung Sik Jin}

Department of Plastic and Reconstructive Surgery, Seoul National University College of Medicine, 101 Daehak-ro, Jongno-gu, Seoul 03080, Korea

E-mail: usj1011@snu.ac.kr

Received February 15, 2015 / Revised April 16, 2015 / Accepted August 4, 2015 quently transported to the emergency room. The patient had multiple facial skeletal injuries with significant malocclusion on physical examination. Computed tomography revealed a bilateral mandibular condylar process fracture and a mandibular parasymphyseal fracture. The patient was taken to the operating room, and under general anesthesia, the mandibular fractures were exposed via an intraoral incision. The mandibular parasymphysis was openly reduced and internally fixated using titanium plates. Most of the dentition consisted of dental implants (Fig. 1). Because of this, we felt arch bars were not appropriate in this patient. Instead, screws and wires were used for intermaxillary fixation.

Seven months post-surgery, the patient underwent a second operation due to trismus and malocclusion. After a bilateral sagittal split ramus osteotomy, precise occlusion was achieved using a wafer. Upon internal fixation of the mandible, intermaxillary fixation was again performed using screws and wires.

Unfortunately, the intermaxillary fixation screws were unable to provide sufficient rigidity, and the patient again developed malocclusion with an anterior open bite (Fig. 2). Hence, the patient underwent a third operation, at seven months after the second operation. After bilateral sagittal split ramus osteotomy, proper 
occlusion was obtained using a prefabricated wafer. In place of the intermaxillary fixation screws, two pairs of Y-shaped plates were used to anchor the intermaxillary fixation wires, which provided more strength and rigidity than the one-point fixation of intermaxillary screws.

The heads of Y-shaped plates were fixed with two 6-mm screws to the maxillary and mandibular alveoli (1 pair each), avoiding the inferior alveolar nerve in the mandible. The tails of Y-shaped plates were bent and exposed to the intraoral space through buccal mucosa. Wires were looped thru the holes and anchored with appropriate tension. Four intermaxillary wires were placed to enhance stability.

Thirty days post-surgery, the wires were replaced with a rubber band (Fig. 3). There were no signs or symptoms of infection or wound problems around the passage of Y-shaped plates through the mucosa, and the plates remained rigidly fixed. The occlusion

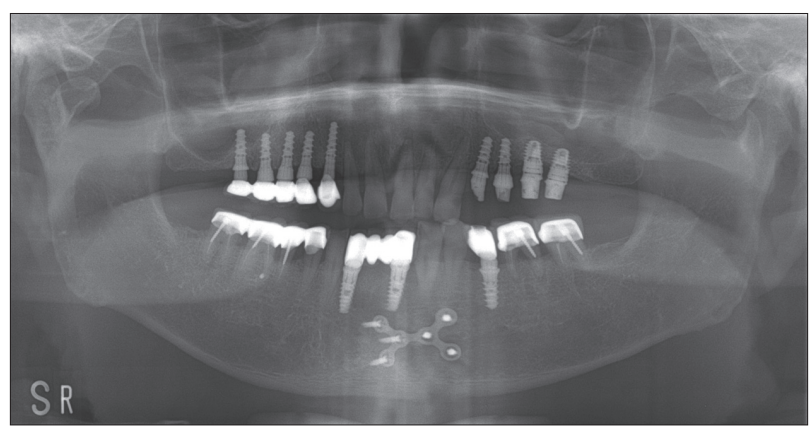

Fig. 1. Postoperative panorama view after the first operation. Most of the dentition consisted of dental implants.

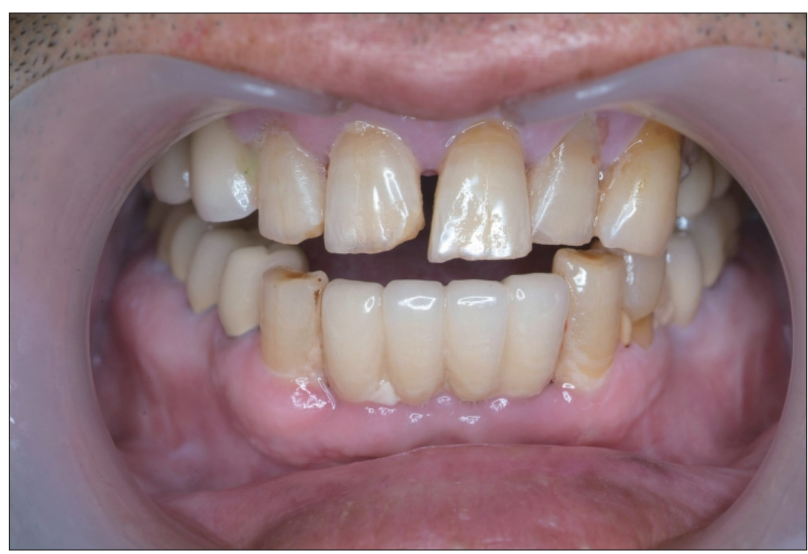

Fig. 2. Occlusal view prior to the third operation shows an anterior open bite. continued to be proper (Fig. 4).

Ninety days later, the Y-shaped plates were removed under local anesthesia (Fig. 5). At the latest follow-up (15 months after the third operation), the occlusion continues to be well maintained.

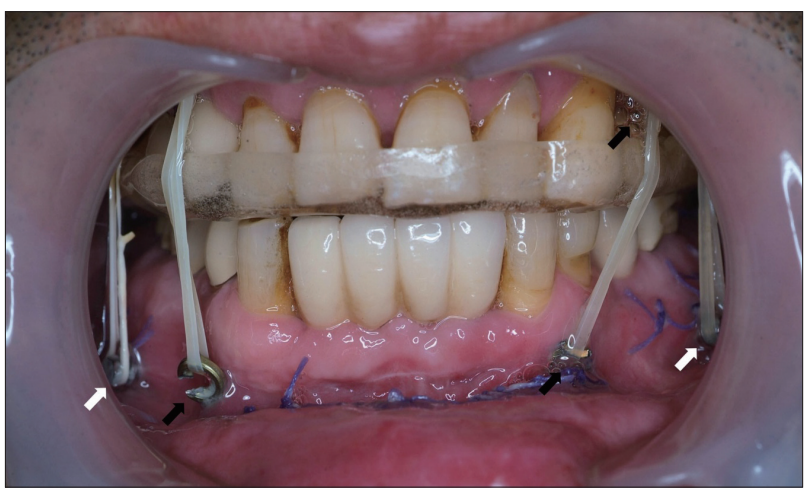

Fig. 3. Occlusal view at 30 days after the third operation demonstrates maintenance of proper occlusion. Black arrows indicate exposed tails of Y-shaped plates. White arrows indicate intermaxillary fixation screws.

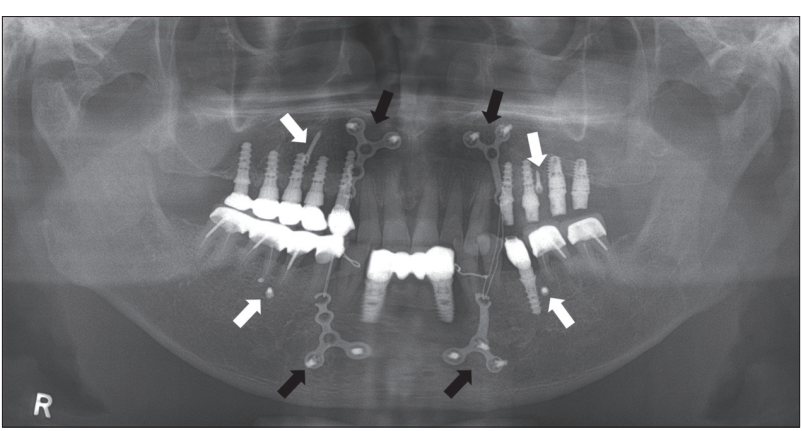

Fig. 4. Panorama view at 3 days after the third operation shows the Y-shaped plates. Black arrows indicate exposed tails of Y-shaped plates. White arrows indicate intermaxillary fixation screws.

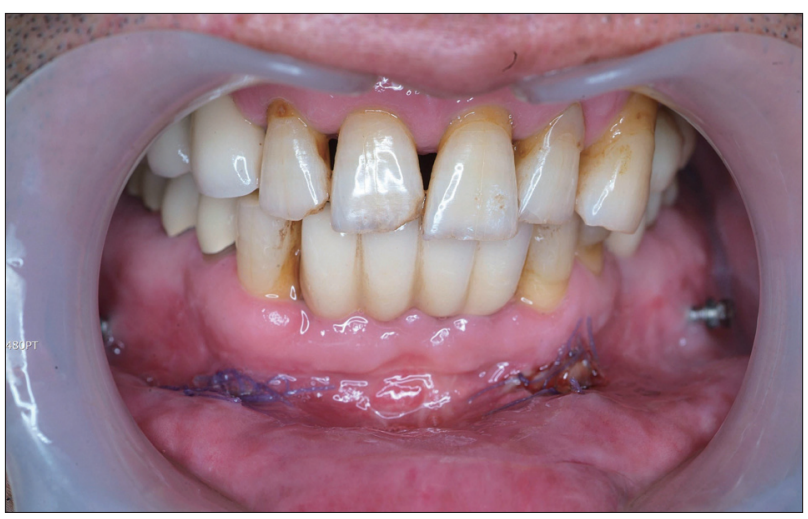

Fig. 5. Occlusal view at 5 days after plate removal. Occlusion continues to be proper, and the mucosal wound is healing well. 


\section{DISCUSSION}

Numerous techniques have been previously described for the management of maxillo-mandibular fractures. Presently, arch bars and wires are the most popular techniques for reducing the maxilla and mandible fixation in proper occlusion. However, this method has several disadvantages. It is associated with a number of complications. Considerable time is needed for placing the arch bar. Maintenance of gingival hygeine is a laborious task, and handling of wires is associated with a high risk of needle stick injury to the surgeon [1].

The use of intermaxillary fixation screws is a common alternate method of intermaxillary fixation. This technique decreases operative time and minimizes the risk of needle stick injuries. In comparison to arch bars, the intermaxillary load is not distributed along the whole of the maxillary and mandibular alveoli. This is associated with problems such as loosening of screws during the postoperative period and damage to dental roots [2].

In patients with poor dentition, neither of the above two methods are suitable. Traditionally, intermaxillary fixation in edentulous patients has been obtained by wiring or rigid fixation of dentures, fabrication of Gunning splints, or by the use of external biphasic pin fixation or halo devices [3]. In our case, an arch bar could not be used for intermaxillary fixation because most of the dentition consisted of dental implants. In addition, intermaxillary fixation screws were unable to provide rigid fixation. Hence, Yshaped plates were needed to provide a solid anchoring platform to provide allow adequate tension between the upper and lower jaw bones. Because of the larger surface area of mucosal disruption from the plates, the risk of infection is higher than for other methods. Thus, close monitoring of gingival and mucosal health is a necessary aspect of postoperative management. Moreover, a second surgery is necessary to remove the plate. Despite these potential problems, we believe that using plate anchors for intermaxillary fixation should be considered for patients in whom conventional approaches are inappropriate, or as in our case, have failed.

In summary, we present a case of repeated intermaxillary fixation failure in a patient weak alveolar rigidity secondary to multiple dental implants. Because single-point fixation screws were not strong enough to maintain proper occlusion, we have used Yshaped plates to provide more rigid anchoring points for the intermaxillary wires. Two pairs of plates were used in our patient, but the number of plates can vary according to the clinical context.

\section{REFERENCES}

1. de Queiroz SB. Modification of arch bars used for intermaxillary fixation in oral and maxillofacial surgery. Int J Oral Maxillofac Surg 2013;42:481-2.

2. Rai A, Datarkar A, Borle RM. Are maxillomandibular fixation screws a better option than Erich arch bars in achieving maxillomandibular fixation? A randomized clinical study. J Oral Maxillofac Surg 2011;69:3015-8.

3. Wolfe SA, Lovaas M, McCafferty LR. Use of a miniplate to provide intermaxillary fixation in the edentulous patient. J Craniomaxillofac Surg 1989;17:31-3. 